\title{
TITLE:
}

\section{MICRODIFFERENTIAL EQUATIONS WITH ISOTROPIC INTERSECTIONS}

$\operatorname{AUTHOR}(\mathrm{S})$ :

UCHIKOSHI, KEISUKE

\section{CITATION:}

UCHIKOSHI, KEISUKE. MICRODIFFERENTIAL EQUATIONS WITH ISOTROPIC INTERSECTIONS. 数理解析研究所講究録 1996, 937: 110-116

ISSUE DATE:

1996-02

URL:

http://hdl.handle.net/2433/60042

RIGHT: 


\title{
MICRODIFFERENTIAL EQUATIONS WITH ISOTROPIC INTERSECTIONS
}

\author{
KEISUKE UCHIKOSHI \\ Department of Mathematics, \\ National Defense Academy
}

\begin{abstract}
For some weakly hyperbolic microdifferential equations we show that the propagation of the singularity is decided by the symplectic structure of their characteristic varieties.
\end{abstract}

Although the propagation of the singularity for weakly hyperbolic operators has been studied by many authors, the detailed results are restricted to two cases: The case of regular involutive characteristics and of non-involutive characteristics. For the first case, it is well-known that the singularity propagates along the integral manifold defined by the symplectic structure of the characteristic variety, and in the second case that the two bicharacteristic strips of the operator interfere each other (See $[8,15,19]$ for the first case and $[1,2,4,5,9,10,11,13,14,16]$ for the second case). We study a new problem including both these two cases. It will turn out that the propagation is completely decided by the symplectic structure of the characteristic variety, also in our framework. This result contains both the above theories. We next remark the fact that it is usual to assume some restrictive conditions for the lower order terms (especially in distribution theory), even for the simpler cases mentioned above. To avoid complicated calculation, we shall assume such an auxiliary condition. However, it is never more restrictive (and sometimes more general) compared with the usual assumptions in the above simpler theories. At last we remark that for this purpose we shall study a theory of some non-local integral operators. The prototype can be found in [6]. The property of the parametrices of microhyperbolic operators was investigated in [7], and we can give a more precise result for the parametrices, in our situation.

Now we give some notations. Let $x^{*}=(0 ; 0, \cdots, 0, \sqrt{-1}) \in \sqrt{-1} \mathbf{T}^{*} \mathbf{R}^{n}$ and $\zeta^{\circ}=$ $(0, \cdots, 0,1) \in \mathbf{R}^{n}(n \in \mathbf{N}=\{1,2,3, \cdots\}) . \mathcal{E}$ (resp. $\left.\mathcal{C}\right)$ denotes the sheaf of microdifferential operators (resp. of microfunctions) on $\sqrt{-1} \mathbf{S}^{*} \mathbf{R}^{n}$, and let $\mathcal{E}_{x^{*}}(j)=\left\{A \in \mathcal{E}_{x^{*}} ;\right.$ ord $A \leq$ $j\}, j \in \mathbf{Z}$. If $A(x, D) \in \mathcal{E}_{x^{*}}{ }^{(j)}$, we denote by $\sigma(A)$ (and sometimes also by $A(x, \xi)$ ) the complete symbol of $A(x, D)$, where $(x, \xi)$ is a homogeneous symplectic coordinate system. Here $D=\partial / \partial x$, as is usual in hyperfunction theory. Conversely if the complete symbol $A(x, \xi)$ of a microdifferential operator is given, we denote this operator by $A(x, D)$. If $A(x, \xi) \sim \sum_{i \leq j} A_{i}(x, \xi)$, where each $A_{i}$ is homogeneous in $\xi$ of degree $i$, we define 
$\sigma_{i}(A)=A_{i}(x, \xi)$. Of course this notation depends on the choice of $(x, \xi)$, except for the principal symbol. If $A(x, D) \in \mathcal{E}_{x^{*}}$, we define

$$
A(\boldsymbol{x}, D)^{j}=\overbrace{A(\boldsymbol{x}, D) \cdots A(\boldsymbol{x}, D)}^{j \text {-times }}, j \in \mathbf{Z}_{+} \cdot
$$

If $A(x, D)$ is elliptic, we define $A(x, D)^{-j}=\left(A^{-1}(x, D)\right)^{j}$. We define $x^{\prime}, x^{\prime \prime} \in \mathbf{R}^{n-1}$ by $\boldsymbol{x}=\left(\boldsymbol{x}_{1}, \boldsymbol{x}^{\prime}\right)=\left(\boldsymbol{x}^{\prime \prime}, \boldsymbol{x}_{n}\right) \in \mathbf{R}^{n}$.

Let $(x, \xi)$ be a homogeneous symplectic coordinate system. We assume that $P_{0}(x, \xi), \cdots, P_{\ell}(x, \xi)(2 \leq \ell \leq n-1)$ are real holomorphic functions defined in a neighborhood of $x^{*}$. We assume that $P_{0}$ is (resp. $P_{1}, \cdots, P_{l}$ are) homogeneous in $\xi$ of degree 0 (resp. 1). We also assume that

$$
\begin{cases}P_{j}\left(x^{*}\right)=0, & 0 \leq j \leq \ell, \\ \left\{P_{i}, P_{j}\right\}=0, & 1 \leq i, j \leq \ell, \\ \left\{P_{0}, P_{1}\right\}\left(x^{*}\right) \neq 0, & \\ \left\{P_{0}, P_{j}\right\}=0, & 2 \leq j \leq \ell, \\ \left(d P_{1} \wedge \cdots \wedge d P_{\ell} \wedge \sum_{1 \leq j \leq n} \xi_{j} d x_{j}\right)\left(x^{*}\right) \neq 0\end{cases}
$$

For instance, $\left(P_{0}, P_{1}, \cdots, P_{\ell}\right)=\left(x_{1}, \xi_{1}, \cdots, \xi_{\ell}\right)$ satisfies (1). Let $m_{0}, m_{1}, \cdots, m_{\ell} \in \mathbf{Z}_{+}=$ $\{0,1,2, \cdots\}$ satisfy $m_{0} \leq m_{1}$. Let $m_{2}+m_{3}+\cdots+m_{\ell}=m^{\prime}$ and $m_{1}+m^{\prime}=m$. We define

$$
\mathcal{E}_{\Lambda}=\left\{A(x, D) \in \mathcal{E}_{x^{*}}{ }^{\left(m^{\prime}\right)} ; \sigma_{m^{\prime}}(A) \text { can be divided by } P_{2}^{m_{2}} P_{3}^{m_{3}} \ldots P_{\ell}^{m_{\ell}}\right\}
$$

and for $A(x, D) \in \mathcal{E}_{\Lambda}$ we define

$$
\sigma_{\Lambda}(A)=P_{2}^{-m_{2}} P_{3}^{-m_{3}} \cdots P_{\ell}^{-m_{\ell}} \sigma_{m^{\prime}}(A) .
$$

We always assume the following condition:

A1

$$
\left\{\begin{array}{l}
P(x, D) \in \mathcal{E}_{x^{*}}^{(m)}, \\
P(x, D)=\sum_{\substack{0 \leq j \leq m_{0} \\
0 \leq k \leq m_{1}-m_{0}}} \exists P_{j, k}(x, D)\left(P_{0}(x, D) P_{1}(x, D)\right)^{j} P_{1}(x, D)^{k} \\
\text { each } P_{j, k} \text { belongs to } \mathcal{E}_{\Lambda} \text { and } \sigma_{\Lambda}\left(P_{m_{0}, m_{1}-m_{0}}\right)=1
\end{array}\right.
$$

Let us define

$$
\begin{aligned}
I_{P}(s)= & \sum_{0 \leq j \leq m_{0}} \sigma_{\Lambda}\left(P_{j, m_{1}-m_{0}}\right)\left(x^{*}\right)\left(\left\{P_{1}, P_{0}\right\}\left(x^{*}\right)\right)^{j-m_{0}} \\
& \times s^{j}(s+1)(s+2) \cdots\left(s+m_{1}-m_{0}\right)
\end{aligned}
$$

We assume that $P(x, D)$ satisfies either

$$
s \in \mathbf{Z}_{+} \Longrightarrow I_{P}(s) \neq 0
$$


or

$$
\mathbf{A 2}^{\prime} \quad s \in\left\{-m_{1}+m_{0}-1,-m_{1}+m_{0}-2, \cdots\right\} \Longrightarrow I_{P}(s) \neq 0 .
$$

It is easy to see that these conditions are invariant under QCT (=quantized contact transformation), defined by [12]. Precisely speaking, let $\kappa_{0}$ be a real homogeneous symplectic transformation defined in a neighborhood of $x^{*}$ satisfying $\kappa_{0}\left(x^{*}\right)=x^{*}$, and let $\kappa: \mathcal{E}_{x^{*}} \longrightarrow \mathcal{E}_{x^{*}}$ be an associated QCT $\left((1)\right.$ is invariant for $\left.\kappa_{0}\right)$. Let $\tilde{P}_{j}=\kappa_{0}^{*}\left(P_{j}\right)$, and define $\tilde{\mathcal{E}}_{\Lambda}$ and $\tilde{\sigma}_{\Lambda}$ for $\tilde{P}_{0}, \cdots, \tilde{P}_{\ell}$ (instead of $P_{0}, \cdots, P_{\ell}$ ), as above. Then we have $\kappa\left(\mathcal{E}_{\Lambda}\right)=\tilde{\mathcal{E}}_{\Lambda}$ and $\tilde{\sigma}_{\Lambda}(\tilde{A})=\kappa_{0}^{*}\left(\sigma_{\Lambda}(A)\right), A \in \mathcal{E}_{\Lambda}$. Furthermore, $P(x, D)$ satisfies $\mathbf{A 1}$ if, and only if, $\tilde{P}(x, D)$ satisfies the same condition for $\tilde{\mathcal{E}}_{\Lambda}$ and $\tilde{\sigma}_{\Lambda}$. For such $P(x, D)$, we can define $\tilde{I}_{\tilde{P}}(s)$ similarly for $\tilde{P}(x, D)$. Since we have $I_{P}(s)=\tilde{I}_{\tilde{P}}(s), \mathbf{A 2}$ and $\mathbf{A 2}$ are invariant for $\kappa$.

To give the main theorem, we prepare the following

Definition 1. (i) Let $\lambda_{1}: \mathbf{R} \longrightarrow \mathbf{R}$ be a strictly increasing continuous function satisfying $\lambda_{1}(0)=0$. Let $\lambda_{2}: \mathbf{R}^{\ell-1} \longrightarrow \mathbf{R}$ be a $C^{1}$ function satisfying $\lambda_{2}(0, \cdots, 0)=0$ and $\partial_{x_{j}} \lambda_{2}\left(x_{2}, \cdots, x_{\ell}\right)>0,2 \leq j \leq \ell$.

(ii) We denote by $\mathcal{O}$ the sheaf of holomorphic functions. Let $S(x, \xi), S^{\prime}(x, \xi) \in \mathcal{O}_{x^{*}}$ and let $P_{j}^{\prime}(x, \xi), Q_{j}(x, \xi) \in \mathcal{O}_{x^{*}}, 1 \leq j \leq n$, be a homogeneous symplectic coordinate system around $x^{*}$ such that

$$
\begin{array}{ll}
S\left(x^{*}\right), S^{\prime}\left(x^{*}\right) \neq 0 & \\
P_{j}^{\prime}\left(x^{*}\right)=\sqrt{-1} \delta_{j n}, & 1 \leq j \leq n, \\
Q_{j}\left(x^{*}\right)=0, & 1 \leq j \leq n, \\
P_{0}=S Q_{1}, P_{j}=S^{\prime} P_{j}^{\prime}, & 1 \leq j \leq \ell,
\end{array}
$$

where $S, S^{\prime}, Q_{j}(1 \leq j \leq n)$ (resp. $P_{j}^{\prime}(1 \leq j \leq n)$ ) are homogeneous in $\xi$ of degree 0 (resp 1). Note that we can choose such functions.

(iii) Let $\overline{\boldsymbol{\omega}} \subset \sqrt{-1} \mathbf{S}^{*} \mathbf{R}^{n}$ be a (fixed) small neighborhood of $\boldsymbol{x}^{*}$. For any open neighbourhood $\omega \subset \bar{\omega}$ of $x^{*}$, we define

$$
\begin{aligned}
& \omega_{1}=\left\{(x, \xi) \infty \in \omega ; \lambda_{2}\left(Q_{2}, \cdots, Q_{\ell}\right) \geq 0,\left|P_{j}^{\prime} / P_{n}^{\prime}\right| \leq \lambda_{1}\left(\left|Q_{1}\right|\right), 1 \leq j \leq \ell\right\} \\
& \omega_{2}=\left\{(x, \xi) \infty \in \omega ; \lambda_{2}\left(Q_{2}, \cdots, Q_{\ell}\right) \geq \lambda_{1}\left(\left|Q_{1}\right|\right)\right\} .
\end{aligned}
$$

We define $\mathcal{C}_{j}=\underset{\omega}{\lim } \Gamma_{\omega_{j}}\left(\omega, \mathcal{C}_{\mathbf{R}^{n}}\right)$ for $j=1,2$.

The main result is the following

Theorem 1. (i) If $P(x, D)$ satisfies $\mathbf{A 1}$ and $\mathbf{A 2}$, then $P(x, D): \mathcal{C}_{1} \longrightarrow \mathcal{C}_{1}$ is surjective.

(ii) If $P(x, D)$ satisfies $\mathbf{A 1}$ and $\mathbf{A 2}{ }^{\prime}$, then $P(x, D): \mathcal{C}_{2} \longrightarrow \mathcal{C}_{2}$ is injective.

Example. Let $P(x, D)=x_{1} D_{1} \cdots D_{\ell} . P$ satisfies $\mathbf{A 1}$ and $\mathbf{A 2}$ for $S=S^{\prime}=1, P_{j}^{\prime}=$ $\xi_{j}, Q_{j}=x_{j}(1 \leq j \leq n)$. We define

$$
\begin{aligned}
b_{j} & =\left\{(x, \xi) \infty \in \sqrt{-1} \mathbf{T}^{*} \mathbf{R}^{n} ; x_{\hat{j}}=0, \xi=\sqrt{-1} \zeta^{o}\right\}, \quad 1 \leq j \leq \ell, \\
b_{1}^{ \pm} & =\left\{(x, \xi) \infty \in b_{1} ; \pm x_{1} \geq 0\right\}
\end{aligned}
$$


where $x_{\hat{j}}=\left(x_{1}, \stackrel{\hat{j}}{\cdot}, x_{n}\right)$. It is easy to see that $u \in \mathcal{C}_{x^{*}}$ satisfies $P u=0$ if, and only if, it is written in the form

$$
u=\operatorname{sp}\left(H\left(x_{1}\right) \exists u_{0}\left(x_{\hat{1}}\right)+\sum_{1 \leq j \leq \ell} \exists u_{j}\left(x_{\hat{j}}\right)\right) .
$$

Here sp $f$ denotes the singularity spectrum of a hyperfunction $f, H$ is the Heaviside function, and $u_{j} \in\left(\mathcal{B}_{\mathbf{R}^{n-1}}\right)_{0}, 0 \leq j \leq \ell$. If $u \neq 0$ (at $\left.x^{*}\right)$, then sp $u_{j} \neq 0$ for some $j$, and supp $u$ must contain at least one of $b_{1}^{+}, b_{1}^{-}, b_{2}, \cdots, b_{\ell}$. It follows that no non-trivial solution belong to $\mathcal{C}_{2}$ (See the figure below). Theorem 1 generalizes this fact.

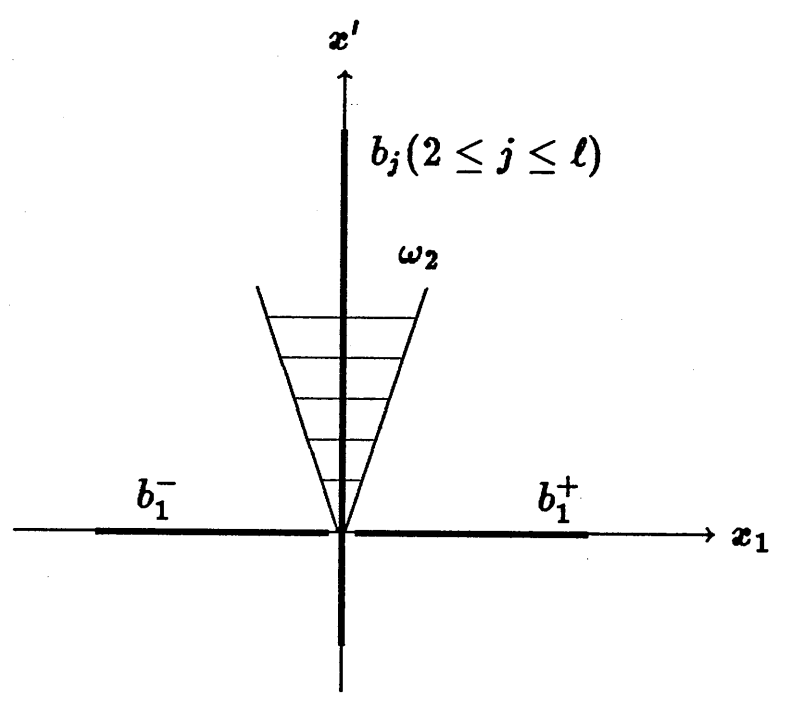

Figure

Remark. If $m_{0}=0$, then $P$ is of regular involutive type, and if $m_{2}=\cdots=m_{\ell}=0$ to the contrary, $P$ is of non-involutive type. In the first case, we do not assume any conditions for the lower order terms. In this case Theorem 1 is well-known under some assumptions for the lower order terms (See $[8,19]$ ). [15] considered this case without such assumptions in the second microlocal category. On the other hand, in the second case our assumption A1 is the usual Levi condition for Fuchsian operators, and $I_{P}(s)$ is also the usual indicial polynomial. In this case Theorem 1 is well-known precisely in this form. However, from now on we always assume that $m_{0}, \cdots, m_{\ell} \geq 1$. Note that this is no restriction. For instance, to prove (i) of Theorem 1 it suffices to show the surjectivity of $P^{\prime}(x, D)=$ $P(x, D) x_{1} D_{1} \cdots D_{\ell}$, instead of $P(x, D)$. By this modification each of $m_{0}, \cdots, m_{\ell}$ increases by one, and if $P$ satisfies $\mathbf{A 1}$ and $\mathbf{A 2}$ then $P^{\prime}$ satisfies the same condition. Similarly, to prove the injectivity of $P(x, D)$, we may consider $D_{\ell} \cdots D_{1} x_{1} P(x, D)$ instead.

In [17] another generalization of Fuchsian operators is investigated. For the sake of simplicity, let the principal symbol of $A(x, D) \in \mathcal{E}_{x^{*}}{ }^{(m)}$ satisfy

$$
\sigma=A_{0}^{m_{0}} A_{1}^{m_{1}} \cdots A_{\ell}^{m_{\ell}}
$$


where $A_{0}$ is (resp. $A_{1}, \cdots, A_{\ell}$ are) homogeneous in $\xi$ of degree 0 (resp. 1), and we assume

$$
\begin{cases}A_{j}\left(x^{*}\right)=0, & 0 \leq j \leq \ell \\ \left\{A_{0}, A_{j}\right\} \neq 0, & 1 \leq j \leq \ell\end{cases}
$$

instead of (1). In [17], under some additional conditions the propagation of the singularities is investigated for such operators. Of course the result is not the same, due to the difference of the symplectic structure of the characteristic variety. Roughly speaking, the singularity of the solutions for such $A(x, D)$ does not propagate along the bicharacteristic strip of $A_{0}$, in some sense (See [17] ). Anyway, non-local integral operators enable us to give clear results on the propagation of the singularities, for various types of weakly hyperbolic operators.

We next explain a non-local operator theory necessary for the proof of Theorem 1. Let $\left(Q_{1}, \cdots Q_{n}, P_{1}^{\prime}, \cdots P_{n}^{\prime}\right)$ be the coordinate system mentioned in Definition 1. From now on we denote this system simply by $(x, \xi)$. We may also assume $P_{0}=x_{1}, P_{j}=\xi_{j}, 1 \leq j \leq \ell$, neglecting elliptic symbols. For the reader's convenience, we rewrite the notations in this coordinate system. $\mathcal{E}_{\Lambda}=\left\{A(x, D) \in \mathcal{E}_{x^{*}{ }^{*}}{ }^{\left(m^{\prime}\right)} ; \sigma_{m^{\prime}}(A)\right.$ can be divided by $\left.\xi_{2}{ }^{m_{2}} \cdots \xi_{\ell}{ }^{m_{\ell}}\right\}$, and for $A(x, D) \in \mathcal{E}_{\Lambda}$ we define $\sigma_{\Lambda}(A)=\xi_{2}{ }^{-m_{2}} \xi_{3}{ }^{-m_{3}} \ldots$

$\xi_{\ell}{ }^{-m_{\ell}} \sigma_{m^{\prime}}(A)$. We always assume the following condition:

$$
\mathbf{A 1}^{\text {bis }} \quad\left\{\begin{array}{l}
P(x, D) \in \mathcal{E}_{x^{*}}^{(m)}, \\
P(x, D)=\sum_{\substack{0 \leq j \leq m_{0} \\
0 \leq k \leq m_{1}-m_{0}}} \exists P_{j, k}(x, D)\left(x_{1} D_{1}\right)^{j} D_{1}^{k}, \\
\text { each } P_{j, k} \text { belongs to } \mathcal{E}_{\Lambda} \text { and } \sigma_{\Lambda}\left(P_{m_{0}, m_{1}-m_{0}}\right)=1 .
\end{array}\right.
$$

We define

$$
I_{P}(s)=\sum_{0 \leq j \leq m_{0}} \sigma_{\Lambda}\left(P_{j, m_{1}-m_{0}}\right)\left(x^{*}\right) s^{j}(s+1)(s+2) \cdots\left(s+m_{1}-m_{0}\right) .
$$

We assume that $P(x, D)$ satisfies either

$$
\text { A2 }^{\text {bis }} \quad s \in \mathbf{Z}_{+} \Longrightarrow I_{P}(s) \neq 0
$$

or

$$
\mathbf{A 2}^{\prime}{ }^{\text {bis }} \quad s \in\left\{-m_{1}+m_{0}-1,-m_{1}+m_{0}-2, \cdots\right\} \Longrightarrow I_{P}(s) \neq 0 .
$$

Let $\overline{\boldsymbol{\omega}} \subset \sqrt{-1} \mathbf{S}^{*} \mathbf{R}^{n}$ be a (fixed) small neighborhood of $x^{*}$. For any open neighbourhood $\boldsymbol{\omega} \subset \overline{\boldsymbol{\omega}}$ of $\boldsymbol{x}^{*}$, we define

$$
\begin{aligned}
& \omega_{1}=\left\{(x, \xi) \infty \in \omega ; \lambda_{2}\left(x_{2}, \cdots, x_{\ell}\right) \geq 0,\left|\xi_{j} / \xi_{n}\right| \leq \lambda_{1}\left(\left|x_{1}\right|\right), 1 \leq j \leq \ell\right\} \\
& \omega_{2}=\left\{(x, \xi) \infty \in \omega ; \lambda_{2}\left(x_{2}, \cdots, x_{\ell}\right) \geq \lambda_{1}\left(\left|x_{1}\right|\right)\right\}
\end{aligned}
$$

and we define $\mathcal{C}_{j}=\underset{\omega}{\lim } \Gamma_{\omega_{j}}\left(\omega, \mathcal{C}_{\mathbf{R}^{n}}\right)$ for $j=1,2$.

We next define two non-local operator classes. 
Definition 2. We denote by $\mathcal{E}_{1}$ the set of germs $k(x, y) \in\left(\mathcal{C}_{\mathrm{R}^{2 n}}\right)_{\left(x^{*},-x^{*}\right)}$ which satisfy

$$
\begin{gathered}
\operatorname{supp} k(x, y) \subset\left\{(x, y ; \xi, \eta) \infty \in \sqrt{-1} \mathbf{S}^{*} \mathbf{R}^{2 n} ; 0 \leq \exists \theta_{1}, \cdots, \exists \theta_{\ell} \leq 1,\right. \\
\\
y_{1}=\theta_{1} x_{1}, \xi_{1}+\theta_{1} \eta_{1}=0 \\
y_{j} \leq x_{j}, \xi_{j}+\theta_{j} \eta_{j}=0,2 \leq j \leq \ell \\
\left.y_{j}=x_{j}, \xi_{j}+\eta_{j}=0, \ell+1 \leq j \leq n\right\}
\end{gathered}
$$

and $\mathcal{E}_{2}$ by $\mathcal{E}_{2}=\left\{k(-y,-x) ; k(x, y) \in \mathcal{E}_{1}\right\}$.

If $A \subset \sqrt{-1} \mathbf{S}^{*} \mathbf{R}^{2 n} \backslash\left(\mathbf{R}^{n} \times \sqrt{-1} \mathbf{S}^{*} \mathbf{R}^{n}\right)$ and $B \subset \sqrt{-1} \mathbf{S}^{*} \mathbf{R}^{n}$, then we define $A \circ B \subset$ $\sqrt{-1} \mathbf{S}^{*} \mathbf{R}^{n}$ by

$$
\begin{gathered}
A \circ B=\left\{(x, \xi) \infty \in \sqrt{-1} \mathbf{S}^{*} \mathbf{R}^{n} ; \text { there exists some }(y, \eta) \infty \in B\right. \\
\text { such that }(x, y, \xi,-\eta) \infty \in A\}
\end{gathered}
$$

Of course we have $\mathcal{E}_{x^{*}} \subsetneq \mathcal{L}_{x^{*}} \subsetneq \mathcal{E}_{1}, \mathcal{E}_{2}$, where $\mathcal{L}$ denotes the sheaf of microlocal operators. We explain some important properties of these operator classes.

Proposition 1. (i) Let $j \in\{1,2\}$. If $k_{1}(x, y), k_{2}(x, y) \in \mathcal{E}_{j}$, then $\int k_{2}(x, z) k_{1}(z, y) d z \in$ $\mathcal{C}_{\left(x^{*},-x^{*}\right)}$ is well-defined and belongs to $\mathcal{E}_{j}$. Thus $\mathcal{E}_{j}$ naturally acquires a ring structure with the unit element $\operatorname{sp} \delta(x-y)$.

(ii) Let $j \in\{1,2\}$. If $u(x) \in \mathcal{C}_{j}$ and $k(x, y) \in \mathcal{E}_{j}$, then $\int k(x, y) u(y) d y \in \mathcal{C}_{x^{*}}$ is welldefined and belongs to $\mathcal{C}_{j}$. Thus $\mathcal{C}_{j}$ naturally acquires the structure of a left $\mathcal{E}_{j}$-module. In fact we have

$$
\operatorname{supp}\left(\int k(x, y) u(y) d y\right) \subset \operatorname{supp} k \text { ○ supp } u \text {. }
$$

Note that the integral operators thus defined are not microlocal, i.e., they may increase the singularities of the operands. Now Theorem 1 is a direct consequence of the following Theorem 2. (i) If $P(x, D)$ satisfies $\mathbf{A 1}$ and $\mathbf{A 2}$, then $P(x, D)$ has a right inverse in $\mathcal{E}_{1}$.

(ii) If $P(x, D)$ satisfies $\mathbf{A 1}$ and $\mathbf{A 2} 2^{\prime}$, then $P(x, D)$ has a left inverse in $\mathcal{E}_{2}$.

We can prove (i) of Theorem 2 using a symbol calculation for $\mathcal{E}_{1}$, and (ii) is its direct consequence. See [18] for the details.

\section{REFERENCES}

[1] S. Alinhac, Branching of singularities for a class of hyperbolic operators, Indiana Univ. Math. J. 27 (1978), 1027-1037.

[2] K. Amano, Branching of singularities for degenerate hyperbolic operators and Stokes phenomena, Proc. Japan Acad. 56 (1980), 206-209.

[3] J. M. Bony and P. Schapira, Propagation des singularités analytiques pour les solutions des équations aux dérivées partielles, Ann. Inst. Fourier 26 (1976), 81-140.

[4] A. Bove, J. E. Lewis, and C. Parenti, Propagation of singularities for Fuchsian operators, Lecture Notes in Math., vol. 984, Springer, Berlin-Heidelberg-New York, 1983.

[5] M. Cicognani and L. Zanghirati, Propagation of analytic and Gevrey singularities for operators with non-involutive characteristics, J. Math. Kyoto Univ. 33 (1989), 633-646.

[6] N. Hanges, Parametrices and propagation of singularities for operators with non-involutive characteristics, Indiana Univ. Math. J. 28 (1979), 87-97. 
[7] M. Kashiwara and T. Kawai, Microhyperbolic pseudodifferential operators I, J. Math. Soc. Japan 27 (1975), 359-404.

[8] Lascar R, Propagation des singularités des solutions d'équations pseudo-différentielles à caractéristiques de multiplicités variables, Lecture Notes in Math., vol. 856, Spinger, Berlin-Heidelberg-New York, 1981.

[9] S. Nakane, Propagation of singularities and uniqueness in the Cauchy problem at a class of doubly characteristic points, Comm. Partial Differential Equations 6 (1981), 917-927.

[10] T. Nishitani, Hyperbolic operators with symplectic multiple characteristics, J. Math. Kyoto Univ. 29 (1989), 405-447.

[11] T. Ôaku, A canonical form of a system of microdifferential equations with non-involutory characteristics and branching of singularities, Invent. Math. 65 (1982), 491-525.

[12] M. Sato, T. Kawai, and M. Kashiwara, Microfunctions and pseudo-differential equations,, Lecture Notes in Math., vol. 287, Springer, Berlin-Heidelberg-New York, 1973.

[13] H. Tahara, Fuchsian type equations and Fuchsian hyperbolic equations, Japan. J. Math. 5 (1979), 245-347.

[14] K. Taniguchi and Y. Tozaki, A hyperbolic equation with double characteristics which has a solution with branching singularities, Math. Japon. 25 (1980), 279-300.

[15] N. Tose, On a class of microdifferential equations with involutory double characteristics, J. Fac. Sci., Univ. of Tokyo 33 (1986), 619-634.

[16] K. Uchikoshi, Microdifferential equations with non-involutory characteristics, Comm. Partial Differential Equations 15 (1990), 1-58.

[17] K. Uchikoshi, Microdifferential equations with non-involutory factors, Comm. Partial Differential Equations 18 (1993), 1557-1587.

[18] K. Uchikoshi, Microdifferential equations with isotropic intersections, to appear in Intern. J. Math..

[19] G. A. Uhlman, Pseudo-differential operators with involutive double characteristics, Comm. Partial Differential Equations 2 (1977), 713-779.

HASHIRIMIZU 1-10-20 YOKOSUKA, JAPAN 